\title{
Tai Chi, Qigong and the Treatment of Disease
}

\author{
Robert W McGee* \\ Department of Graduate and Professional Studies in Business, Fayetteville State University, USA \\ *Corresponding author: Robert W McGee, Department of Graduate and Professional Studies in Business, USA
}

\begin{abstract}
ARTICLE INFO
Received: 蔧 February 20, 2021

Published: 慧 March 02, 2021

Citation: Robert W McGee. Tai Chi, Qigong and the Treatment of Disease. Biomed J Sci \& Tech Res 34(2)-2021. BJSTR. MS.ID.005531.

ABSTRACT

Qigong has been around for more than 4,000 years. It contains aspects of martial arts, philosophy, theology, mindfulness, meditation, exercise, and Traditional Chinese Medicine (TCM). It is a subfield of both tai chi and health economics. Practicing qigong exercises on a regular basis can improve health and cause the body to function more efficiently. Thus, it is also a subfield of applied microeconomics in the sense that it increases efficiency. Numerous medical studies have been done on the effect of qigong and tai chi on various health conditions. The present paper presents a brief overview of some recent applications of qigong to the treatment of various diseases.
\end{abstract}

Keywords: Qigong; Tai Chi; Ba Duan Jin; TCM; Traditional Chinese Medicine; Treatment of Disease

\section{Introduction}

Qi (pronounced chee) can be translated as energy. Gong can be translated as work. Thus, qigong can be translated as energy work. The history of qigong goes back thousands of years [1,2]. Qigong exercises have become a part of Traditional Chinese Medicine (TCM), which focuses more on prevention rather than treatment of disease [2-11]. Entire hospitals in China are devoted to using qigong to treat diseases. Several organizations focus much or all their attention either to research on the health benefits of qigong or to teach health qigong to the masses [12-44]. Qigong has both physical and mental components. It is a form of dynamic meditation, in the sense that it involves movement. It also involves breathing and mindfulness. Qigong exercises can be performed from both standing and sitting positions. There are literally thousands of qigong exercises, and medical qigong practitioners often mix and match qigong exercises to customize a training regimen for their patients.

The most popular set of qigong exercises is ba duan jin, a set of eight qigong exercises that cover all the main energy meridians $[45,46]$. Millions of people all over the world perform the ba duan jin exercises daily to maintain and improve health. Performing all eight exercises takes between 10-15 minutes, depending on the speed with which they are performed and the number of repetitions. Of all the qigong exercise sets that exist, the ba duan jin exercises are probably the set that is most prescribed to patients. According to one recent survey of 886 clinical studies of qigong, ba duan jin was studied in 55.5 percent of the cases [47]. However, ba duan jin is not the only set of qigong exercises that medical practitioners prescribe to their patients. Other popular qigong sets include Liu Zi Jue [48], Wu Qin Xi [49,50], Yi Jin Jing [51,52], Da Wu [53], Shi Er Duan Jin [54], Daoyin Yangsheng Gong Shi Er Fa [55], Mawangdui [56], and Taiji Yangsheng Zhang [57]. Much medical research has been conducted in recent years on the use of qigong to treat various diseases [58-61], Tai chi is related to qigong, but it is not qigong [62]. Tai chi also involves dynamic meditation and breathing, if performed properly, but the movements are different than those of qigong, as is the emphasis. Tai chi is also part of TCM and is used in the treatment of various diseases. The present paper provides a brief overview of some of the applications of qigong that have been used in the treatment of various diseases.

\section{Anxiety}

Liu, et al. [63] surveyed the literature and found that qigong exercises had an improvement effect on individuals suffering from anxiety compared to medication. A study by Li, et al. [64] found that qigong, tai chi and yoga exercises had a beneficial effect on COPD patients suffering from anxiety and depression.

\section{Arthritis}

Chen, et al. [65] found that dao yin (qigong) exercises had a beneficial effect on several kinds of arthritis. Marks [66] found that qigong can be used to alleviate longstanding or chronic musculoskeletal pain. Xie, et al. [67] found that qigong exercises 
improved symptoms of ankylosing spondylitis. At least three studies found that baduanjin had a beneficial effect on knee osteoarthritis [68-70].

\section{Attention Deficit}

Duarte, et al. [71] found that "White Ball" qigong was able to improve the attention of adolescents after four weeks of practice.

\section{Autism}

Rodrigues, et al. [72] Found that qigong was able to decrease the severity of sensory, behavioral and language components of autism, and improve sociability, self-control, cognitive and sensory awareness on children and adolescents.

\section{Back Pain}

Chen, et al. [73] found that qigong exercises were beneficial in relieving low back pain. Zou, et al. [74] found that tai chi and qigong exercises were more beneficial at relieving low back pain than yoga.

\section{Blood Pressure}

Liu, et al. [75] found that tai chi and qigong were effective in reducing systolic and diastolic blood pressure and concluded that they could be effective complementary or alternative therapies for essential hypertension (EH). Park, et al. [76] conducted a four-week study of expert-led qigong, and found a significant reduction in DBP, indicating that qigong can be a safe and effective intervention for patients with prehypertension and stage I hypertension. However, patients doing self-practice qigong showed no significant change in SBP and DBP, indicating that an expert-led qigong program is recommended for blood pressure management.

\section{Cancer}

Numerous studies have been conducted on the effects of tai chi and qigong exercises on the treatment of cancer, cancer fatigue $[77,78]$, pain management $[79,80]$, mindfulness and cognitive impairment [81-84], sleep [85], heart rate variability [86], quality of life [87] and bio sociality [88]. Generally, it was found that tai chi and qigong exercises had a beneficial effect. Some studies examined the effect that tai chi or qigong exercises had on specific types of cancer. Breast cancer patients used tai chi or qigong exercises to alleviate sleep problems [89] and pain [90]. Zhang, et al. did a study on the effects of qigong exercises on cognitive impairment in breast cancer patients [91]. Another study examined the effectiveness of exercised-based interventions in reducing fatigue, dyspnea and depression in lung cancer patients [92]. The effects of qigong or tai chi exercises on prostate cancer patients were also examined $[93,94]$.

\section{Chronic Fatigue Syndrome, Cognitive Impairment and COPD}

Other studies involved chronic fatigue syndrome [95], cognitive impairment [96-101], COPD [102].

\section{Covid-19}

Qigong exercises tended to have a beneficial effect on Covid-19 patients [103-105].

\section{Depression}

Numerous studies have been done on the effect of qigong or tai chi exercises on depressions. Results have generally shown to be positive [106-115].

\section{Fibromyalgia}

A study of the effects of regular qigong exercises on fibromyalgia patients found that their mean score differences for pain, sleep quality, chronic fatigue, anxiety, depression and fibromyalgia impact were all significantly better than those of the control group $(\mathrm{p}<0.05)[116]$.

\section{Frailty}

A study of frailty in elderly patients found that a steady regimen of ba duan jin exercises improved physical and cognitive function, reduced fall risk, improved psychological well-being and reversed frailty status [117].

\section{Heart Disease}

Mao, et al. [118] found that the regular practice of ba duan jin exercises in STEMI patients could alleviate the adverse LV remodeling that is associated with beneficial energy metabolism adaptation, inflammation, curbing, and extracellular matrix organization adjustment.

\section{Immune System}

Studies have found that the regular practice of qigong exercises can strengthen the immune system $[119,120]$.

\section{Parkinson's Disease}

Several studies found that the practice of qigong exercises can have a positive effect motor function, walking ability and balance for individuals having Parkinson's disease [121-126].

\section{Pulmonary Disease}

Several studies have examined the effect that qigong or tai chi exercises have on patients with pulmonary diseases of various kinds. The results have generally been positive [127-131].

\section{Quality of Life}

Several studies have found that the regular practice of qigong exercises have a positive effect on the quality of life for patients who have a wide range of diseases or illnesses [132-135].

\section{Schizophrenia}

Meditation-based mind-body therapies such as tai chi and qigong might have a beneficial effect on the negative symptoms of schizophrenia, but more research is needed [136]. 


\section{Stress}

Bischoff, et al. [137] found that yoga and qigong might be an effective method for reducing stress in healthcare professionals.

\section{Stroke}

Some studies have found that qigong exercises can improve the physical and mental state of patients who have had a stroke, could reduce the effects of sleep and mental disorders and improve muscle function [138-141].

\section{Substance Abuse}

Liu, et al. [142] found that qigong exercises could have a beneficial effect on anxiety among drug abusers. However, there were weaknesses in their study, so more research is needed on this topic.

\section{Unilateral Vocal Fold Paralysis}

Tang, et al. [143] found that Liuzijue qigong exercises could improve the vocal function of patients with unilateral vocal fold paralysis, but that there was not a significant difference between the liuzijue patients and those who received the conventional treatment for the ailment, which might lead one to conclude that the qigong exercises could be used as an alternative to conventional treatment.

\section{Conclusion}

Many studies have found that tai chi and qigong exercises can have a beneficial effect for patients having a wide range of ailments. In some cases, the results from qigong and tai chi approaches were significantly better than those of other approaches, while in other cases, the results were comparable, leading one to conclude that tai chi and qigong exercises might be used as alternative therapies in some cases, depending on the ailment.

\section{Conflict of Interest}

There is no conflict of interest.

\section{Author Bio}

Robert W. McGee is a professor in the business school of Fayetteville State University, USA. He holds 13 earned doctorates from universities in the United States and four European countries, and has published more than 60 books, as well as more than 700 scholarly papers. He holds black belt/duan rank in eight martial arts, including tai chi, and is a world champion in tai chi (both Sun and Yang styles), taekwondo, kung-fu, karate and qigong.

\section{References}

1. Jahnke, Roger (1997) The Healer Within. San Francisco: Harper.

2. Johnson, Jerry Alan (2000) Chinese Medical Qigong Therapy: A Comprehensive Clinical Guide. Pacific Grove, CA: International Institute of Medical Qigong.

3. Frantzis, Bruce (2010) Dragon and Tiger Medical Qigong, Volume 1. Fairfax, CA: Energy Arts.
4. Frantzis, Bruce (2014) Dragon and Tiger Medical Qigong Volume 2: Qi Cultivation Principles and Exercises. North Atlantic Books.

5. Johnson, Jerry Alan (2005) Chinese Medical Qigong Therapy, Vol. 1: Energetic Anatomy and Physiology. Pacific Grove, CA: International Institute of Medical Qigong.

6. Johnson, Jerry Alan (2005) Chinese Medical Qigong Therapy, Vol. 2: Pacific Grove, CA: International Institute of Medical Qigong.

7. Johnson, Jerry Alan (2002) Chinese Medical Qigong Therapy, Vol. 3: Pacific Grove, CA: International Institute of Medical Qigong.

8. Johnson, Jerry Alan (2002) Chinese Medical Qigong Therapy, Vol. 4: Prescription Exercises and Meditations, Treatment of Internal Diseases, Pediatrics, Geriatrics, Gynecology, Neurology, and Energetic Psychology. Pacific Grove, CA: International Institute of Medical Qigong.

9. Johnson, Jerry Alan (2005) Chinese Medical Qigong Therapy, Vol. 5: An Energetic Approach to Oncology. Pacific Grove, CA: International Institute of Medical Qigong.

10. Jahnke, Roger (2002) The Healing Promise of Qi. New York: Contemporary Books, a division of McGraw-Hill.

11. Jingwei li, Zhu Jianping (2014) The Illustrated Handbook of Chinese Qigong Forms from the Ancient Texts. London \& Philadelphia: Singing Dragon.

12. American Academy for Medical Qigong.

13. American Qigong Association.

14. American Tai Chi and Qigong Association.

15. British Health Qigong Association.

16. Center for Taiji \& Qigong Studies.

17. The Chi Center.

18. Energy Arts.

19. European Sports Qigong Association.

20. Flowing Zen Qigong \& Tai Chi.

21. Holden Qigong.

22. Hong Kong Tai Chi \& Qigong.

23. The Institute for Rehabilitative Qigong \& Tai Chi.

24. Institute of Integral Qigong and Tai Chi.

25. Instituto Qigong Barcelona.

26. International Health Qigong Federation.

27. International Medical Tai Chi and Qigong Association.

28. International Qi Gong Association.

29. Lin Housheng's Qigong.

30. National Qigong Association.

31. Qi Journal.

32. Qigong Awareness.

33. Qigong and Daoist Training Center.

34. Qigong Global Summit.

35. Qigong Institute.

36. The Qigong Research Society.

37. Robert Peng.

38. Spring Forest Qigong.

39. Tai Chi Healthways.

40. Tai Chi, Qigong \& Feng Shui Institute. 
41. Tse Qigong Centre.

42. United States Health Qigong Association.

43. World Tai Chi \& Qigong Day.

\section{YOQI Yoga \& Qigong.}

45. (2008) Chinese Health Qigong Association. Ba Duan Jin. Beijing: Foreign Languages Press.

46. McGee, Robert W (2020) Ba Duan Jin as a Treatment for Physical Ailments: A Bibliography of Recent Medical Research, Fayetteville State University, Broadwell College of Business and Economics, Studies in the Economics of Qigong 3(2020).

47. Zhang YP, Hu RX, Han M, Lai BY, Liang SB, et al. (2020) Evidence Base of Clinical Studies on Qi Gong: A Bibliometric Analysis. Complementary therapies in medicine 50: 102392.

48. (2007) Chinese Health Qigong Association. Liu Zi Jue. Beijing, China.

49. (2008) Chinese Health Qigong Association. Wu Qin Xi. Beijing China.

50. McGee, Robert W (2020) Wu Qin Xi as a Treatment for Physical Ailments: A Bibliography of Recent Medical Research, Fayetteville State University, Broadwell College of Business and Economics, Studies in the Economics of Qigong 4(2020).

51. (2009) Chinese Health Qigong Association. Yi Jin Jing. Beijing, China.

52. McGee, Robert W (2020) The Use of Yi Jin Jing to Treat Illness: A Summary of Three Studies, Fayetteville State University, Broadwell College of Business and Economics, Studies in the Economics of Qigong $5(2020)$

53. (2014) Chinese Health Qigong Association. Da Wu. Beijing: Foreign Languages Press.

54. (2014) Chinese Health Qigong Association. Shi Er Duan Jin. Beijing: Foreign Languages Press.

55. (2014) Chinese Health Qigong Association. Daoyin Yangsheng Gong Shi Er Fa. Beijing: Foreign Languages Press.

56. (2014) Chinese Health Qigong Association. Mawanhdui Daoyin Shu. Beijing: Foreign Languages Press.

57. (2014) Chinese Health Qigong Association. Taiji Yangsheng Zhang. Beijing: Foreign Languages Press.

58. McGee, Robert W (2020) A Bibliography of Recent Medical Research on Qigong, Fayetteville State University, Broadwell College of Business and Economics, Studies in the Economics of Qigong 2(2020).

59. PubMed.gov. National Library of Medicine, National Center for Biotechnology Information, National Institute of Health.

60. Qigong and Energy Medicine Database.

61. The Qigong Research Society.

62. Douglas, Bill (1999) The Complete Idiot's Guide to T'ai Chi \& QiGong. New York: Alpha Books, a division of Macmillan General Reference.

63. Liu F, Cui J, Liu X, Chen KW, Chen X, Li R (2020) The effect of tai chi and Qigong exercise on depression and anxiety of individuals with substance use disorders: a systematic review and meta-analysis. BMC complementary medicine and therapies 20(1): 161.

64. Li Z, Liu S, Wang L, Smith L (2019) Mind-Body Exercise for Anxiety and Depression in COPD Patients: A Systematic Review and Meta-Analysis. International journal of environmental research and public health 17(1): 22.

65. Chen X, Cui J, Li R, Norton R, Park J, et al. (2019) Dao Yin (a.k.a. Qigong): Origin, Development, Potential Mechanisms, and Clinical Applications. Evidence-based complementary and alternative medicine: eCAM 2019: 3705120.

66. Marks R (2019) Qigong and Musculoskeletal Pain. Current rheumatology reports 21(11): 59 .
67. Xie Y, Guo F, Lu Y, Guo Y, Wei G, et al. (2019) A 12-week Baduanjin Qigong exercise improves symptoms of ankylosing spondylitis: A randomized controlled trial. Complementary therapies in clinical practice 36: 113119.

68. Ye J, Simpson MW, Liu Y, Lin W, Zhong W. et al. (2020) The Effects of Baduanjin Qigong on Postural Stability, Proprioception, and Symptoms of Patients With Knee Osteoarthritis: A Randomized Controlled Trial. Frontiers in medicine 6: 307.

69. Ye J, Zheng Q, Zou L, Yu Q Veronese N, et al. (2020) Mindful Exercise (Baduanjin) as an Adjuvant Treatment for Older Adults (60 Years Old and Over) of Knee Osteoarthritis: A Randomized Controlled Trial. Evidencebased complementary and alternative medicine: eCAM 2020: 9869161.

70. Zeng ZP, Liu YB, Fang J, Liu Y, Luo J, et al. (2020) Effects of Baduanjin exercise for knee osteoarthritis: A systematic review and meta-analysis. Complementary therapies in medicine 48: 102279.

71. Duarte L, Gonçalves M, Mendes P, Matos LC, Greten HJ, et al. (2020) Can Qigong improve attention in adolescents? A prospective randomised controlled trial. Journal of bodywork and movement therapies 24(1): 175-181.

72. Rodrigues JM, Mestre M, Fredes LI (2019) Qigong in the treatment of children with autism spectrum disorder: A systematic review. J Integr Med 17(4): 250-260.

73. Chen X, Cui J, Li R, Norton R, Park J, et al. (2019) Dao Yin (a.k.a. Qigong): Origin, Development, Potential Mechanisms, and Clinical Applications. Evidence-based complementary and alternative medicine: eCAM 2019: 3705120.

74. Zou L, Zhang Y, Yang L, Loprinzi PD, Yeung AS, et al. (2019) Are Mindful Exercises Safe and Beneficial for Treating Chronic Lower Back Pain? A Systematic Review and Meta-Analysis of Randomized Controlled Trials. Journal of clinical medicine 8(5): 628.

75. Liu D, Yi L, Sheng M, Wang G, Zou Y (2020) The Efficacy of Tai Chi and Qigong Exercises on Blood Pressure and Blood Levels of Nitric Oxide and Endothelin-1 in Patients with Essential Hypertension: A Systematic Review and Meta-Analysis of Randomized Controlled Trials. Evidencebased complementary and alternative medicine: eCAM 2020(1): 3267971.

76. Park JE, Yang CS, Jung SY, Kim AR, Lee M, et al. (2019) Exploring the effects of expert-led qigong and self-practice qigong on blood pressure. Integrative medicine research 8(4): 271-272.

77. Arring NM, Barton DL, Brooks T, Zick SM (2019) Integrative Therapies for Cancer-Related Fatigue. Cancer journal (Sudbury, Mass.) 25(5): 349356.

78. Sowada KM (2019) Qigong: Benefits for Survivors Coping with CancerRelated Fatigue. Clinical journal of oncology nursing 23(5): 465-469.

79. Deng G (2019) Integrative Medicine Therapies for Pain Management in Cancer Patients. Cancer journal (Sudbury, Mass.) 25(5): 343-348.

80. Maindet C, Burnod A, Minello C, George B, Allano G, et al. (2019) Strategies of complementary and integrative therapies in cancer-related pain-attaining exhaustive cancer pain management. Supportive care in cancer : official journal of the Multinational Association of Supportive Care in Cancer 27(12): 3119-3132.

81. Campbell KL, Zadravec K, Bland KA, Chesley E, Wolf F, et al. (2020) The Effect of Exercise on Cancer-Related Cognitive Impairment and Applications for Physical Therapy: Systematic Review of Randomized Controlled Trials. Physical therapy 100(3): 523-542.

82. Cheng TC, Lee YH, Mar CL, Huang WT, Chang YP, et al. (2020) The Health Promoting Mindfulness or Qigong Educational Programs for Beneficial Lifestyle Changes of Cancer Survivors. Journal of cancer education: the official journal of the American Association for Cancer Education 35(4): 743-750.

83. Ford CG, Vowles KE, Smith BW, Kinney AY (2020) Mindfulness and Meditative Movement Interventions for Men Living with Cancer: A Meta- 
analysis. Annals of behavioral medicine: a publication of the Society of Behavioral Medicine 54(5): 360-373.

84. Oberoi D, Piedalue KL, Pirbhai H, Guirguis S, Santa Mina D, et al. (2020) Factors related to dropout in integrative oncology clinical trials: interim analysis of an ongoing comparative effectiveness trial of mindfulnessbased cancer recovery and Tai chi/Qigong for cancer health (The MATCH study). BMC research notes 13(1): 342.

85. Garland SN, Mahon K, Irwin MR (2019) Integrative Approaches for Sleep Health in Cancer Survivors. Cancer journal (Sudbury, Mass.) 25(5): 337342.

86. Larkey L, Kim W, James D, Kishida M, Vizcaino M, et al. (2020) MindBody and Psychosocial Interventions May Similarly Affect Heart Rate Variability Patterns in Cancer Recovery: Implications for a Mechanism of Symptom Improvement. Integrative cancer therapies 19: 1534735420949677.

87. Lin WF, Zhong MF, Zhou QH, Zhang YR, Wang H, et al. (2019) Efficacy of complementary and integrative medicine on health-related quality of life in cancer patients: a systematic review and meta-analysis. Cancer management and research 11: 6663-6680.

88. Li F, Wang C (2020) "A Good Guy" Again: Biosociality in a Cancer Selfhelp Organization. Medical anthropology 1-14.

89. Kreutz C, Schmidt ME, Steindorf K (2019) Effects of physical and mind-body exercise on sleep problems during and after breast cancer treatment: a systematic review and meta-analysis. Breast cancer research and treatment 176(1): 1-15.

90. Osypiuk K, Ligibel J, Giobbie-Hurder A, Vergara-Diaz G, Bonato P, et al. (2020) Qigong Mind-Body Exercise as a Biopsychosocial Therapy for Persistent Post-Surgical Pain in Breast Cancer: A Pilot Study. Integrative cancer therapies 19: 1534735419893766.

91. Zhang Q, Gao X, Liu S, Yu L, Zhu J, et al. (2020) Therapies for cognitive impairment in breast cancer survivors treated with chemotherapy: A protocol for systematic review. Medicine 99(19): e20092.

92. Henshall CL, Allin L, Aveyard H (2019) A Systematic Review and Narrative Synthesis to Explore the Effectiveness of Exercise-Based Interventions in Improving Fatigue, Dyspnea, and Depression in Lung Cancer Survivors. Cancer nursing 42(4): 295-306.

93. Kinney AY, Blair CK, Guest DD, Ani JK, Harding EM, et al. (2019) Biobehavioral effects of Tai Chi Qigong in men with prostate cancer: Study design of a three-arm randomized clinical trial. Contemporary clinical trials communications 16: 100431. H

94. Zuniga KB, Chan JM, Ryan CJ, Kenfield SA (2020) Diet and lifestyle considerations for patients with prostate cancer. Urologic oncology 38(3): 105-117.

95. Xie F, Guan C, Cheng Z, Yao F, You Y, et al. (2020) Effects of the prolong life with nine turn method (Yan Nian Jiu Zhuan) Qigong on patients with chronic fatigue syndrome: study protocol for a randomized controlled trial. Annals of palliative medicine apm 9(5): 19-461.

96. Campbell KL, Zadravec K, Bland KA, Chesley E, Wolf F, et al. (2020) The Effect of Exercise on Cancer-Related Cognitive Impairment and Applications for Physical Therapy: Systematic Review of Randomized Controlled Trials. Physical therapy 100(3): 523-542.

97. Chan J, Deng K, Wu J, Yan J H (2019) Effects of Meditation and Mind-Body Exercises on Older Adults' Cognitive Performance: A Meta-analysis. The Gerontologist 59(6): e782-e790.

98. Ibañez GE, Fennie K, Larkey L (2019) Qigong as a promising mindbody exercise for cognitive functioning: Letter to Editor. Journal of the American Geriatrics Society 67(7): 1533-1534.

99. Wu C, Tang C (2019) Reply to: Qigong as a Promising Mind-Body Exercise for Cognitive Functioning. Journal of the American Geriatrics Society 67(7): 1534-1535

100. Zhang Q Gao X, Liu S, Yu L, Zhu J, et al. (2020) Therapies for cognitive impairment in breast cancer survivors treated with chemotherapy: A protocol for systematic review. Medicine 99(19): e20092.
101. Zou L, Loprinzi PD, Yeung AS, Zeng N, Huang T, et al. (2019) The Beneficial Effects of Mind-Body Exercises for People with Mild Cognitive Impairment: a Systematic Review With Meta-analysis. Archives of physical medicine and rehabilitation 100(8): 1556-1573.

102. Li Z, Liu S, Wang L, Smith L (2019) Mind-Body Exercise for Anxiety and Depression in COPD Patients: A Systematic Review and Meta-Analysis. International journal of environmental research and public health, 17(1): 22

103. Chen JM, Wang ZY, Chen YJ, Ni J (2020) The Application of Eight-Segment Pulmonary Rehabilitation Exercise in People with Coronavirus Disease 2019. Frontiers in physiology 11: 646.

104. Feng F, Tuchman S, Denninger J W, Fricchione GL, Yeung A, et al. (2020) Qigong for the Prevention, Treatment, and Rehabilitation of COVID-19 Infection in Older Adults. The American journal of geriatric psychiatry: official journal of the American Association for Geriatric Psychiatry 28(8): 812-819.

105. Zhang S, Zhu Q, Zhan C, Cheng W, Mingfang X, et al. (2020) Acupressure therapy and Liu Zi Jue Qigong for pulmonary function and quality of life in patients with severe novel coronavirus pneumonia (COVID-19): a study protocol for a randomized controlled trial. Trials 21(1): 751.

106. Gill BK, CantR, Lam L,Cooper S, Lou V, etal. (2020) Non-pharmacological depression therapies for older Chinese adults: A systematic review \& meta-analysis. Archives of gerontology and geriatrics 88: 104037.

107. Gouw V, Jiang Y, Seah B, He H, Hong J, et al. (2019) Effectiveness of internal Qigong on quality of life, depressive symptoms and selfefficacy among community-dwelling older adults with chronic disease: A systematic review and meta-analysis. International journal of nursing studies 99: 103378

108. Henshall CL, Allin L, Aveyard H (2019) A Systematic Review and Narrative Synthesis to Explore the Effectiveness of Exercise-Based Interventions in Improving Fatigue, Dyspnea, and Depression in Lung Cancer Survivors. Cancer nursing 42(4): 295-306.

109. Jin X, Wang L, Liu S, Zhu L, Loprinzi PD, et al. (2019) The Impact of Mind-body Exercises on Motor Function, Depressive Symptoms, and Quality of Life in Parkinson's Disease: A Systematic Review and Metaanalysis. International journal of environmental research and public health 17(1): 31.

110. Lee P, Cai S, Lu EY, Ng B, Jensen MP, et al. (2020) Qigong Reduces Depressive Symptoms of Taiwanese Elderly with Chronic Physical Illness: A Randomized Controlled Trial. Journal of alternative and complementary medicine (New York, N.Y.) 26(1): 76-78.

111. Li Z, Liu S, Wang L, Smith L (2019) Mind-Body Exercise for Anxiety and Depression in COPD Patients: A Systematic Review and Meta-Analysis. International journal of environmental research and public health 17(1): 22.

112. Liu F, Cui J, Liu X, Chen KW, Chen X, et al. (2020) The effect of tai chi and Qigong exercise on depression and anxiety of individuals with substance use disorders: a systematic review and meta-analysis. BMC complementary medicine and therapies 20(1): 161.

113. Lu EY, Lee P, Cai S, So W, Ng B, et al. (2020) Qigong for the treatment of depressive symptoms: Preliminary evidence of neurobiological mechanisms. International journal of geriatric psychiatry, 10.1002/ gps.5380.

114. So WWY, Cai S, Yau SY, Tsang HWH (2019) The Neurophysiological and Psychological Mechanisms of Qigong as a Treatment for Depression: A Systematic Review and Meta-Analysis. Front Psychiatry 10: 820.

115. Zaprutko T, Göder R, Rybakowski F, Kus K, Kopciuch D, et al. (2020) Non-pharmacological treatments of inpatients with major depression - The case of Polish (Poznan) and German (Kiel) hospital. Complementary therapies in clinical practice 39: 101129.

116. Sarmento C, Moon S, Pfeifer T, Smirnova IV, Colgrove Y, et al. (2020) The therapeutic efficacy of Qigong exercise on the main symptoms of fibromyalgia: A pilot randomized clinical trial. Integrative medicine research 9(4): 100416 . 
117. Liu X, Seah J, Pang B, Tsao MA, Gu F, et al. (2020) A single-arm feasibility study of community-delivered Baduanjin (Qigong practice of the eight Brocades) training for frail older adults. Pilot and feasibility studies 6: 105.

118. Mao S, Zhang X, Chen M, Wang C, Chen Q et al. (2020) Beneficial Effects of Baduanjin Exercise on Left Ventricular Remodelling in Patients after Acute Myocardial Infarction: an Exploratory Clinical Trial and Proteomic Analysis. Cardiovascular drugs and therapy 10.1007/ s10557-020-07047-0.

119. Chen X, Cui J, Li R, Norton R, Park J, et al. (2019) Dao Yin (a.k.a. Qigong): Origin, Development, Potential Mechanisms, and Clinical Applications. Evidence-based complementary and alternative medicine: eCAM 3705120 .

120. Oh B, Bae K, Lamoury G, Eade T, Boyle F, et al. (2020) The Effects of Ta Chi and Qigong on Immune Responses: A Systematic Review and MetaAnalysis. Medicines (Basel, Switzerland) 7(7): 39.

121. Chen S, Zhang Y, Wang YT, Liu X, Song W, et al. (2020) The effect of Qigong-based therapy on patients with Parkinson's disease: a systematic review and meta-analysis. Clinical rehabilitation 269215520946695.

122. Fidan O, Seyyar GK, Aras B, Colak E, Aras O, et al. (2019) The effect of Tai Chi and Qigong on health-related quality of life in Parkinson's disease: a systematic review and meta-analysis of systematic reviews. International journal of rehabilitation research. Internationale Zeitschrift fur Rehabilitations for schung. Revue internationale de recherches de readaptation 42(3): 196-204.

123. Jin X, Wang L, Liu S, Zhu L, Loprinzi PD, et al. (2019) The Impact of Mind-body Exercises on Motor Function, Depressive Symptoms, and Quality of Life in Parkinson's Disease: A Systematic Review and Metaanalysis. International journal of environmental research and public health 17(1): 31 .

124. Li Z, Zhuang J, Jiang Y, Xiao G, Jie K, et al. (2019) Study protocol for a single-blind randomised controlled trial to evaluate the clinical effects of an Integrated Qigong exercise intervention on freezing of gait in Parkinson's disease. BMJ open 9(9): e028869.

125. Moon S, Sarmento C, Steinbacher M, Smirnova IV, Colgrove Y, et al. (2020) Can Qigong improve non-motor symptoms in people with Parkinson's disease - A pilot randomized controlled trial? Complementary therapies in clinical practice 39: 101169.

126. Tang L, Fang Y, Yin J (2019) The effects of exercise interventions on Parkinson's disease: A Bayesian network meta-analysis. Journal of clinical neuroscience: official journal of the Neurosurgical Society of Australasia 70: 47-54.

127. Chen JM, Wang ZY, Chen YJ, Ni J (2020) The Application of EightSegment Pulmonary Rehabilitation Exercise in People with Coronavirus Disease 2019. Frontiers in physiology 11: 646.

128. Guo C, Xiang G, Xie L, Liu Z, Zhang X (2020) Effects of Tai Chi training on the physical and mental health status in patients with chronic obstructive pulmonary disease: a systematic review and metaanalysis. Journal of thoracic disease 12(3): 504-521.

129. Tong H, Liu Y, Zhu Y, Zhang B, Hu J, et al. (2019) The therapeutic effects of qigong in patients with chronic obstructive pulmonary disease in the stable stage: a meta-analysis. BMC complementary and alternative medicine 19(1): 239.

130. Yu F, Xin M, Liu N, Huang N, Lu J, et al. (2019) The Qigong Wuqinxi for chronic obstructive pulmonary disease: Protocol for a systematic review and meta-analysis. Medicine 98(30): e16633.
131. Zhang S, Zhu Q, Zhan, Cheng W, Mingfang X, et al. (2020) Acupressure therapy and Liu Zi Jue Qigong for pulmonary function and quality of life in patients with severe novel coronavirus pneumonia (COVID-19): a study protocol for a randomized controlled trial. Trials 21(1): 751.

132. Chen CH, Hung KS, Chung YC, Yeh ML (2019) Mind-body interactive qigong improves physical and mental aspects of quality of life in inpatients with stroke: A randomized control study. European journal of cardiovascular nursing: journal of the Working Group on Cardiovascular Nursing of the European Society of Cardiology 18(8): 658-666.

133. Gouw V, Jiang Y, Seah B, He H, Hong J, et al. (2019) Effectiveness of internal Qigong on quality of life, depressive symptoms and selfefficacy among community-dwelling older adults with chronic disease: A systematic review and meta-analysis. International journal of nursing studies 99: 103378.

134. Jin X, Wang L, Liu S, Zhu L, Loprinzi PD, et al. (2019) The Impact of Mind-body Exercises on Motor Function, Depressive Symptoms, and Quality of Life in Parkinson's Disease: A Systematic Review and Metaanalysis. International journal of environmental research and public health 17(1): 31.

135. Lin WF, Zhong MF, Zhou QH, Zhang YR, Wang H, et al. (2019) Efficacy of complementary and integrative medicine on health-related quality of life in cancer patients: a systematic review and meta-analysis. Cancer management and research 11: 6663-6680.

136. Sabe M, Sentissi O, Kaiser S (2019) Meditation-based mind-body therapies for negative symptoms of schizophrenia: Systematic review of randomized controlled trials and meta-analysis. Schizophrenia research 212: 15-25.

137. Bischoff LL, Otto AK, Hold C, Wollesen B (2019) The effect of physical activity interventions on occupational stress for health personnel: A systematic review. International journal of nursing studies 97: 94-104.

138. Chen CH, Hung KS, Chung YC, Yeh ML (2019) Mind-body interactive qigong improves physical and mental aspects of quality of life in inpatients with stroke: A randomized control study. European journal of cardiovascular nursing: journal of the Working Group on Cardiovascular Nursing of the European Society of Cardiology 18(8): 658-666.

139. Dong X, Zhang R, Guo Y, Chen L, Liu Y, et al. (2020) The efficacy of Qigong exercises for post-stroke mental disorders and sleep disorders: Protocol for a systematic review and meta-analysis. Medicine 99(34): e21784.

140. Wang C, Yu L, Yang J, Wang RW, Zheng YN, et al. (2020) Effectiveness of LiuZiJue Qigong versus traditional core stability training for poststroke patients complicated with abnormal trunk postural control: study protocol for a single center randomized controlled trial. Trials 21(1): 254.

141. Zheng Y, Zhang Y, Li H, Qiao L, Fu W, et al. (2021) Comparative effect of Liuzijue Qigong and conventional respiratory training on trunk control ability and respiratory muscle function in patients at an early recovery stage from stroke: a randomized controlled trial. Archives of physical medicine and rehabilitation 102(3): 423-430.

142. Liu F, Cui J, Liu X, Chen KW, Chen X, et al. (2020) The effect of tai chi and Qigong exercise on depression and anxiety of individuals with substance use disorders: a systematic review and meta-analysis. BMC complementary medicine and therapies 20(1): 161.

143. Tang J, Huang W, Chen X, Lin Q, Wang T, et al. (2019) Liuzijue Qigong: A Voice Training Method for Unilateral Vocal Fold Paralysis Patients. The Annals of otology, rhinology, and laryngology 128(7): 654-661. 
ISSN: 2574-1241

DOI: 10.26717/BJSTR.2021.34.005531

Robert W McGee. Biomed J Sci \& Tech Res

(C) (P) This work is licensed under Creative

Submission Link: https://biomedres.us/submit-manuscript.php

$\begin{array}{ll}\text { BIOMEDICAL } & \text { Assets of Publishing with us } \\ \text { RESEARCHES } & \text { - Global archiving of articles } \\ \text { - Immediate, unrestricted online access } \\ \text { - Rigorous Peer Review Process }\end{array}$

